\title{
Grand Challenges in Microalgae Domestication
}

\author{
Eric Maréchal * \\ Laboratoire de Physiologie Cellulaire et Végétale, CNRS, CEA, INRAE, Université Grenoble Alpes, IRIG, CEA Grenoble, \\ Grenoble, France
}

Keywords: microalgae, domestication, algae based bioproducts, lipids, pigments, biofuel, green chemistry

\section{INTRODUCTION}

The term "microalgae" is a practical designation for photosynthetic unicellular cells, embracing organisms from two domains of life, i.e., Bacteria (Cyanobacteria) and various clades of Eukaryota deriving from primary (Archaeplastida) or secondary (e.g., Stramenopile) endosymbiosis events. In spite of this dispersed taxonomic distribution, microalgae share features that make them somehow "alike". Oxygenic photosynthesis derives from a common origin and makes microalgae prominent in trophic networks, as primary producers. They are unicellular or form very small colonies, and their cultivation rely on common methods, with provision of light, $\mathrm{CO}_{2}$, water, and nutrients. Microalgae produce valuable molecules, like glycans, lipids, pigments, proteins, etc. Thus, although inappropriate in botanical or taxonomic sense, the term "microalgae" takes its legitimate meaning in ecology and human industry. This is both a weakness, when trying to transfer knowledge from one organism to another, and a strength, when addressing similar biotechnological questions. The development of a microalgae-based industry has become a societal challenge in the past decade. The climatic emergency and pressure on arable lands make the need for novel carbon-free and sustainable productions each day more urgent. Applications range from food, health, green chemistry to biofuels, with the promise of biomolecules produced from $\mathrm{CO}_{2}$ captured from the atmosphere or carbon-emitting industries. In this context, an "algae sector" has emerged, gathering actors specialized in algae cultivation, harvesting, extraction processes, and biorefinery.

Turning a wild algal strain into an "algal crop", i.e., "domesticating" a microalga, represents a grail, because initial traits of interest may be present, like a relatively high level of oil, carbohydrates, pigments, etc., but the path to an enhanced, reproducible and up-scalable yield is extremely challenging.

Some lessons can be learnt from agriculture and give a novel stimulus to research in the microalgae sector. When one walks in nature, does he or she find wild plants resembling wheat, maize, tomato, sunflower, rapeseed, etc? Crop plants look giant and obese, when compared to their wild counterparts. Furthermore, after harvest, it is rare that cultivated seeds escape and invade uncultivated areas. Plant domestication is therefore focused on productivity and quality, but not on fitness in competition with wild communities. The strong difference between wild and domesticated plants illustrates that gains in yield should be obtainable in other branches of life, keeping in mind that cultivated plants are diploid, whereas the majority of currently cultivated microalgae are haploid.

\section{BASIC LESSONS FROM AGRICULTURE}

The domestication of crop plants started during the Neolithic Period and can be reconstructed based on population genetics studies, the analysis of archeological artifacts and the experimental 
validation of reasonable hypotheses. In this first period of agriculture, the purification of strains by selecting traits of interest and the control of breeding techniques were consciously or unconsciously the strategies of choice.

If we focus on "gene targets" governing traits of interest, a well-known example is the domestication of teosinte into the crop maize. It involved "domestication genes" controlling the growth of lateral stems or formation of naked kernels (Chen et al., 2021). With genetic and agronomic strategies developed in the last century, additional mutations allowed the cultivation of maize virtually in all places in the world, with remarkably high yields. Targeting genes can now be much faster using CRISPR/Cas9-based methods (Lemmon et al., 2018). It is also feasible to produce plants devoid of any foreign DNA by methods collectively called next-generation or new breeding techniques (NBTs) (Holme et al., 2019). Some of the NBTs target genes with a Cas9-coding DNA vector who does not integrate into the plant genome, or even a microinjected Cas9 protein and gRNAs, whereas other NBTs are based on non-targeted random mutations (Holme et al., 2019; Anders et al., 2021). The concept of "gene target" proved determinant in the understanding of some domesticated organisms. The "de novo" domestication of tomato could thus be reconstructed experimentally gene-by-gene (Lemmon et al., 2018). This being stated, when transferred to microalgae, it would be naïve to limit the understanding of domestication to the modification of well-selected genes. Some traits depend on complex interactions, and strategies should also consider regulatory genes controlling broad metabolic, physiological, or developmental processes, combinations of genes and non-targeted multiple random mutations.

Plant domestication also highlights the benefits of features, which cannot be reduced to a gene target approach. In schematic terms, improved traits can emerge from specific "rearrangements" or "duplications of chromosomes". In the simplest example in maize, the crossing of homozygous parent lines can give rise to an heterozygous "hybrid" with superior robustness and productivity, a phenomenon called "heterosis" (Srivastava et al., 2020; Chen et al., 2021). Polyploidy can also "improve" patterns of gene expression (Schaart et al., 2021). On the one hand, it can be a high level of "autopolyploidy", which can be induced by a variety of techniques (Chen et al., 2020). On the other hand, "allopolyploids" deriving from the crossing of very close but distinct species, can give rise to progenies retaining the parent chromosomes and exhibiting higher productivity and robustness. Rapeseed (Brassica napus) is an allotetraploid, containing chromosomes from B. rapa and B. olearacea parents (Mason and Snowdon, 2016). Likewise, common wheat (Triticum aestivun) is a hexaploid combining chromosomes from diverse Triticum and Aegilops species (Parisod and Badaeva, 2020). The improvement of algal strains by developing hybrids, autopolyploids or allopolyploids is currently barely explored.

The last lesson from plant domestication may be that growth, productivity, resistance to diseases, etc., can be addressed by domestication strategies, but the response to abiotic environmental stressors, like drought or nutrient scarcity are more difficult questions (Li et al., 2021).

\section{CHALLENGES IN MICROALGAE DOMESTICATION}

With the notable exceptions of yeast and some fungi, or Chlamydomonas, genetics is poorly developed in microbiology. Due to their unicellular nature, microalgae populate environments (oceans, rivers, soils, snow, ice, extreme habitats, etc.) primarily by mitotic divisions, either in haploid or diploid forms. The majority of microbial organisms are not cultivable (Ding et al., 2014). Strains collected in nature can be purified using a cell sorter or by serial cultivation on petri dishes until obtaining clonal axenic lines. Sometimes it is not possible to separate a microalga from its companion bacteria (Lupette et al., 2016). Only in response to environmental or physiological triggers, do gametogenesis, sexual reproduction and meiosis occur (Lopez et al., 2015). Whereas, breeding and shuffling of natural alleles are major drivers of diversity in plants and animals, they do not seem to play this role in microalgae. Genetic diversity relies on genomic mutation rates and transfers of genetic material within and between species. A bias in the microbiological approach of domestication, compared to agriculture, is that the majority of strategies aims at developing an improved clonal strain, and disregard more global approaches with multiple series of mutant lines, which could be crossed, combined and improved.

A first challenge is to pursue the exploration of biodiversity. This includes efforts to resolve the question of non-cultivable strains. Some qualitative traits like the capacity to produce a biomolecule of interest may serve as a starting point for domestication attempts; nevertheless, the capacity to grow fast and produce biomass should be considered an important initial property for further consideration.

A second challenge is the lack of sequenced genomes and the difficulty to genetically transform many non-model and emerging model species. Let us cover the biodiversity of microalgae pointing to some examples of current efforts on "algal crop" models.

- In Cyanobacteria, spirulina (Arthrospira platensis) is "technically" haploid. It contains a rich equipment of restriction enzymes, and methods to efficiently transform this popular Cyanobacterial crop have been made available only recently (Jeamton et al., 2017; Dehghani et al., 2018).

- In Eukaryota, the Archaeplastida comprise three lineages: Green Algae, Red Algae, and Glaucophyta. The Green Alga genetic model is Chlamydomonas reinhardtii, with a genome sequenced two decades ago (Blaby et al., 2014; Lopez et al., 2015). Its life cycle relies mainly on haploid asexual divisions; sexual reproduction can be controlled in vitro (Wilson, 2008). Nuclear and chloroplastic transformations are possible and multiple methods have been developed for gene editing (Ghribi et al., 2020). It is thus considered as a model for synthetic biology (Scaife et al., 2015). In spite of these advantages, its limited biomass and productivity does not make C. reinhardtii a real crop (Butler et al., 2020). Chlorella and Dunaliella species have life cycles close to that of $C$. 
reinhardtii. Chlorella are maintained in haploid form, and it is difficult to know whether sexual reproduction could be obtained. Genomic data have been made available recently for a few strains (Wu et al., 2019) and transformation mediated by Agrobacterium tumefaciens is possible (Cha et al., 2012; Sharma et al., 2021). Dunalliella has a main haploid cycle, but sexual reproduction is known. Draft genomic data are available (Polle et al., 2017) and nuclear and chloroplastic transformations have been validated recently (Dehghani et al., 2017; Norzagaray-Valenzuela et al., 2018; Bahador et al., 2019; Song et al., 2019). Technically both Chlorella and Dunaliella could be amenable to intensive genetic engineering. Scenedesmus and Haematococcus species are Green Algae producing pluri-nuclear cells, with four to eight haploid nuclei, in the course of cell fission. Genomes of S. obliquus (Nag Dasgupta et al., 2018; Astafyeva et al., 2020) and H. pluvialis (Luo et al., 2019; Morimoto et al., 2020) are available. Transformation of $S$. obliquus via A. tumefasciens is feasible (Suttangkakul et al., 2019). Both nuclear and chloroplastic transformations have been described for $H$. pluvialis (Yuan et al., 2019; Wang et al., 2020; Cui et al., 2021). Finally, Charophyta are Green Algae close to Embryophyta, propagating by haploid asexual division; sexual reproduction is known. Genomic data are available for the Charophyta model Klebsormidium (Hori et al., 2014), and transformation and gene editing have been obtained in a Closterium species (Abe et al., 2011). In Red Algae, Galdieria sulphuraria is considered for its cultivation at high temperature and acidity. Its small genome has been known for nearly two decades (Barbier et al., 2005), and genetic engineering is possible (Fujiwara et al., 2019).

- In photosynthetic Stramenopiles, a branch of Eukaryota deriving from a secondary endosymbiosis, the diatom Phaeodactylum and the eustigmatophytes Nannochloropsis/Microchloropsis species are crop models studied by multiple groups worldwide. Robust genomic data (Bowler et al., 2008; Vieler et al., 2012) and tools for genetic engineering are available; multiple examples show the power of gene editing to improve traits and domesticate these lines (Siaut et al., 2007; De Riso et al., 2009; Kilian et al., 2011; Cao et al., 2012; Daboussi et al., 2014; Dolch et al., 2017; Poliner et al., 2018a; Nobusawa et al., 2019; Billey et al., 2021). Remarkably, whereas eustigmatophytes are maintained in haploid form, the vegetative cells of diatoms are diploid.

In the above listed microalgae, strategies developed on multiple gene targets rely on recent technological developments. Progresses in non-GMO plant domestication also inspire the search for methods allowing the transient expression of Cas9 and gRNAs, for instance, by an episomal DNA, removed after lifting the vector selection pressure, to generate strains without any foreign DNA (Poliner et al., 2018b; Sharma et al., 2018; Moosburner et al., 2020).

A third challenge is to control meiosis, gametogenesis, and sexual reproduction. Conventional genetics could boost targeted strategies, by allowing allelic rearrangements. Major breakthroughs could be anticipated if sexual reproduction could be controlled in routine in Chlorella, Dunaliella, Scenedesmus, Klebsormidium, Phaeodactylum etc. We need to keep in mind that in some algal species, the existence of mating types may limit the capacity to develop homozygous diploids requiring the development of self-mating lines (Kariyawasam et al., 2019).

A fourth challenge lies in the development of systems allowing the control of chromosomic combinations and duplication. Heterosis relies directly on the capacity to obtain heterozygous hybrids from homozygous lines. Diatoms have diploid vegetative cells allowing the exploration of this property. The development of autopolyploids from haploid cells has been attempted in pioneering experiments by treatments of Chlamydomonas with molecules blocking cell division, like colchicine (Wetherell and Krauss, 1956) or colcemide (Kwak et al., 2017; Kariyawasam et al., 2019). In diploid Chlamydomonas lines, the lipid yield was improved (Kwak et al., 2017). Concerning allopolyploidy, sexual reproduction between distinct parental species needs to occur. The genome of the diatom Fistullifera solaris derives from two distinct parental species (Tanaka et al., 2015). F. solaris is oleaginous, suggesting that allopolyploidy may have a positive impact on productivity in diatoms as well. Future work on cell fusion may help circumventing the need for a sexual reproduction, within or between species, to further explore this potential.

Eventually, a last challenge concerns random mutagenesis and in vivo directed evolution approaches (Crook et al., 2016) as a way to domesticate microalgae (Pourmir and Johannes, 2012; Rossoni and Weber, 2019). Such strategies require excellent screening methods for selection of improved lines. They also need sequencing efforts to characterize mutants and comprehend the relation between genomic changes and gained properties.

In conclusion, microalgae domestication is in its infancy. The exploration of biodiversity needs to be pursued. Given the development of enabling methods in an increasing number of microalgal groups, there is no doubt that the coming decade will be marked by fascinating results.

\section{AUTHOR CONTRIBUTIONS}

EM has conceived and written the manuscript.

\section{FUNDING}

EM was supported by the Agence Nationale de la Recherche (Alpalga ANR-20-CE02-0020, Oceanomics ANR-11-BTBR-0008, GlycoAlps ANR-15-IDEX-02, GRAL Labex ANR-10-LABEX04, and EUR CBS ANR-17-EURE-0003) and Institut Carnot 3BCAR. 


\section{REFERENCES}

Abe, J., Hori, S., Tsuchikane, Y., Kitao, N., Kato, M., and Sekimoto, H. (2011). Stable nuclear transformation of the closterium peracerosum-strigosumlittorale complex. Plant Cell Physiol. 52, 1676-1685. doi: 10.1093/pcp/pcr103

Anders, S., Cowling, W., Pareek, A., Gupta, K. J., Singla-Pareek, S. L., and Foyer, C. H. (2021). Gaining acceptance of novel plant breeding technologies. Trends Plant Sci. 26, 575-587. doi: 10.1016/j.tplants.2021.03.004

Astafyeva, Y., Alawi, M., Indenbirken, D., Danso, D., Grundhoff, A., Hanelt, D., et al. (2020). Draft genome sequence of the green alga Scenedesmus acuminatus SAG 38.81. Microbiol. Resour. Announc. 9:e01278-19. doi: 10.1128/MRA.01278-19

Bahador, E., Einali, A., Azizian-Shermeh, O., and Sangtarash, M. H. (2019). Metabolic responses of the green microalga Dunaliella salina to silver nanoparticles-induced oxidative stress in the presence of salicylic acid treatment. Aquat. Toxicol. 217:105356. doi: 10.1016/j.aquatox.2019.105356

Barbier, G., Oesterhelt, C., Larson, M. D., Halgren, R. G., Wilkerson, C., Garavito, R. M., et al. (2005). Comparative genomics of two closely related unicellular thermo-acidophilic red algae, Galdieria sulphuraria and Cyanidioschyzon merolae, reveals the molecular basis of the metabolic flexibility of Galdieria sulphuraria and significant differences in carbohydrate metabolism of both algae. Plant Physiol. 137, 460-474. doi: 10.1104/pp.104.051169

Billey, E., Magneschi, L., Leterme, S., Bedhomme, M., Andres-Robin, A., Poulet, L., et al. (2021). Characterization of the bubblegum acyl-CoA synthetase of Microchloropsis gaditana. Plant Physiol. 185, 815-835. doi: 10.1093/plphys/kiaa110

Blaby, I. K., Blaby-Haas, C. E., Tourasse, N., Hom, E. F., Lopez, D., Aksoy, M., et al. (2014). The chlamydomonas genome project: a decade on. Trends Plant Sci. 19, 672-680. doi: 10.1016/j.tplants.2014.05.008

Bowler, C., Allen, A. E., Badger, J. H., Grimwood, J., Jabbari, K., Kuo, A., et al. (2008). The phaeodactylum genome reveals the evolutionary history of diatom genomes. Nature 456, 239-244. doi: 10.1038/nature07410

Butler, T., Kapoore, R. V., and Vaidyanathan, S. (2020). Phaeodactylum tricornutum: a diatom cell factory. Trends Biotechnol. 38, 606-622. doi: 10.1016/j.tibtech.2019.12.023

Cao, S., Zhang, X., Ye, N., Fan, X., Mou, S., Xu, D., et al. (2012). Evaluation of putative internal reference genes for gene expression normalization in Nannochloropsis sp. by quantitative real-time RT-PCR. Biochem. Biophys. Res. Commun. 424, 118-123. doi: 10.1016/j.bbrc.2012.06.086

Cha, T. S., Yee, W., and Aziz, A. (2012). Assessment of factors affecting Agrobacterium-mediated genetic transformation of the unicellular green alga, Chlorella vulgaris. World J. Microbiol. Biotechnol. 28, 1771-1779. doi: 10.1007/s11274-011-0991-0

Chen, J. T., Coate, J. E., and Meru, G. (2020). Editorial: artificial polyploidy in plants. Front. Plant Sci. 11:621849. doi: 10.3389/fpls.2020.621849

Chen, Q., Li, W., Tan, L., and Tian, F. (2021). Harnessing knowledge from maize and rice domestication for new crop breeding. Mol. Plant 14, 9-26. doi: $10.1016 /$ j.molp.2020.12.006

Crook, N., Abatemarco, J., Sun, J., Wagner, J. M., Schmitz, A., and Alper, H. S. (2016). In vivo continuous evolution of genes and pathways in yeast. Nat. Commun. 7:13051. doi: 10.1038/ncomms13051

Cui, H., Zhao, C., Xu, W., Zhang, H., Hang, W., Zhu, X., et al. (2021). Characterization of type-2 diacylglycerol acyltransferases in Haematococcus lacustris reveals their functions and engineering potential in triacylglycerol biosynthesis. BMC Plant Biol. 21:20. doi: 10.1186/s12870-02002794-6

Daboussi, F., Leduc, S., Marechal, A., Dubois, G., Guyot, V., PerezMichaut, C., et al. (2014). Genome engineering empowers the diatom Phaeodactylum tricornutum for biotechnology. Nat. Commun. 5:3831. doi: $10.1038 /$ ncomms4831

De Riso, V., Raniello, R., Maumus, F., Rogato, A., Bowler, C., and Falciatore, A. (2009). Gene silencing in the marine diatom Phaeodactylum tricornutum. Nucleic Acids Res. 37:e96. doi: 10.1093/nar/gkp448

Dehghani, J., Adibkia, K., Movafeghi, A., Barzegari, A., Pourseif, M. M., Maleki Kakelar, H., et al. (2018). Stable transformation of Spirulina (Arthrospira) platensis: a promising microalga for production of edible vaccines. Appl. Microbiol. Biotechnol. 102, 9267-9278. doi: 10.1007/s00253-0189296-7
Dehghani, J., Movafeghi, A., Barzegari, A., and Barar, J. (2017). Efficient and stable transformation of Dunaliella pseudosalina by 3 strains of Agrobacterium tumefaciens. Bioimpacts 7, 247-254. doi: 10.15171/bi.2017.29

Ding, X., Cao, C. C., and Sun, X. (2014). Intrinsic correlation of oligonucleotides: a novel genomic signature for metagenome analysis. J. Theor. Biol. 353, 9-18. doi: 10.1016/j.jtbi.2014.02.039

Dolch, L. J., Rak, C., Perin, G., Tourcier, G., Broughton, R., Leterrier, M., et al. (2017). A palmitic acid elongase affects eicosapentaenoic acid and plastidial monogalactosyldiacylglycerol levels in nannochloropsis. Plant Physiol. 173, 742-759. doi: 10.1104/pp.16.01420

Fujiwara, T., Hirooka, S., Mukai, M., Ohbayashi, R., Kanesaki, Y., Watanabe, S., et al. (2019). Integration of a Galdieria plasma membrane sugar transporter enables heterotrophic growth of the obligate photoautotrophic red alga Cynanidioschyzon merolae. Plant Direct 3:e0134. doi: 10.1002/pld3.134

Ghribi, M., Nouemssi, S. B., Meddeb-Mouelhi, F., and Desgagne-Penix, I. (2020). Genome editing by CRISPR-Cas: a game change in the genetic manipulation of Chlamydomonas. Life 10:295. doi: 10.3390/life10110295

Holme, I. B., Gregersen, P. L., and Brinch-Pedersen, H. (2019). Induced genetic variation in crop plants by random or targeted mutagenesis: convergence and differences. Front. Plant Sci. 10:1468. doi: 10.3389/fpls.2019.01468

Hori, K., Maruyama, F., Fujisawa, T., Togashi, T., Yamamoto, N., Seo, M., et al. (2014). Klebsormidium flaccidum genome reveals primary factors for plant terrestrial adaptation. Nat. Commun. 5:3978. doi: 10.1038/ncomms4978

Jeamton, W., Dulsawat, S., Tanticharoen, M., Vonshak, A., and Cheevadhanarak, S. (2017). Overcoming intrinsic restriction enzyme barriers enhances transformation efficiency in Arthrospira platensis C1. Plant Cell Physiol. 58, 822-830. doi: 10.1093/pcp/pcx016

Kariyawasam, T., Joo, S., Goodenough, U., and Lee, J. H. (2019). Novel approaches for generating and manipulating diploid strains of Chlamydomonas reinhardtii. Algae 34, 35-43. doi: 10.4490/algae.2019.34.2.25

Kilian, O., Benemann, C. S., Niyogi, K. K., and Vick, B. (2011). High-efficiency homologous recombination in the oil-producing alga Nannochloropsis sp. Proc. Natl. Acad. Sci. U.S.A. 108, 21265-21269. doi: 10.1073/pnas.1105861108

Kwak, M., Park, W. K., Shin, S. E., Koh, H. G., Lee, B., Jeong, B. R., et al. (2017). Improvement of biomass and lipid yield under stress conditions by using diploid strains of Chlamydomonas reinhardtii. Algal Res. Biomass Biofuels Bioproducts 26, 180-189. doi: 10.1016/j.algal.2017.07.027

Lemmon, Z. H., Reem, N. T., Dalrymple, J., Soyk, S., Swartwood, K. E., Rodriguez-Leal, D., et al. (2018). Rapid improvement of domestication traits in an orphan crop by genome editing. Nat. Plants 4, 766-770. doi: 10.1038/s41477-018-0259-x

Li, S., Zhang, C., Li, J., Yan, L., Wang, N., and Xia, L. (2021). Present and future prospects for wheat improvement through genome editing and advanced technologies. Plant Commun. 2:100211. doi: 10.1016/j.xplc.2021.100211

Lopez, D., Hamaji, T., Kropat, J., De Hoff, P., Morselli, M., Rubbi, L., et al. (2015). Dynamic changes in the transcriptome and methylome of Chlamydomonas reinhardtii throughout its life cycle. Plant Physiol. 169, 2730-2743. doi: 10.1104/pp.15.00861

Luo, Q., Bian, C., Tao, M., Huang, Y., Zheng, Y., Lv, Y., et al. (2019). Genome and transcriptome sequencing of the astaxanthin-producing green microalga, Haematococcus pluvialis. Genome Biol. Evol. 11, 166-173. doi: 10.1093/gbe/evy263

Lupette, J., Lami, R., Krasovec, M., Grimsley, N., Moreau, H., Piganeau, G., et al. (2016). Marinobacter dominates the bacterial community of the Ostreococcus tauri phycosphere in culture. Front. Microbiol. 7:1414. doi: $10.3389 /$ fmicb.2016.01414

Mason, A. S., and Snowdon, R. J. (2016). Oilseed rape: learning about ancient and recent polyploid evolution from a recent crop species. Plant Biol. 18, 883-892. doi: $10.1111 /$ plb.12462

Moosburner, M. A., Gholami, P., Mccarthy, J. K., Tan, M., Bielinski, V. A., and Allen, A. E. (2020). Multiplexed knockouts in the model diatom phaeodactylum by episomal delivery of a selectable Cas9. Front. Microbiol. 11:5. doi: 10.3389/fmicb.2020.00005

Morimoto, D., Yoshida, T., and Sawayama, S. (2020). Draft genome sequence of the astaxanthin-producing microalga Haematococcus lacustris strain NIES-144. Microbiol. Resour. Announc. 9:e00128-20. doi: 10.1128/MRA.00128-20

Nag Dasgupta, C., Nayaka, S., Toppo, K., Singh, A. K., Deshpande, U., and Mohapatra, A. (2018). Draft genome sequence and detailed characterization 
of biofuel production by oleaginous microalga Scenedesmus quadricauda LWG002611. Biotechnol. Biofuels 11, 308. doi: 10.1186/s13068-018$1308-4$

Nobusawa, T., Yamakawa-Ayukawa, K., Saito, F., Nomura, S., Takami, A., and Ohta, H. (2019). A homolog of Arabidopsis SDP1 lipase in Nannochloropsis is involved in degradation of de novo-synthesized triacylglycerols in the endoplasmic reticulum. Biochim. Biophys. Acta 1864, 1185-1193. doi: 10.1016/j.bbalip.2019.05.013

Norzagaray-Valenzuela, C. D., German-Baez, L. J., Valdez-Flores, M. A., Hernandez-Verdugo, S., Shelton, L. M., and Valdez-Ortiz, A. (2018). Establishment of an efficient genetic transformation method in Dunaliella tertiolecta mediated by Agrobacterium tumefaciens. J. Microbiol. Methods 150, 9-17. doi: 10.1016/j.mimet.2018.05.010

Parisod, C., and Badaeva, E. D. (2020). Chromosome restructuring among hybridizing wild wheats. New Phytol. 226, 1263-1273. doi: 10.1111/nph.16415

Poliner, E., Farre, E. M., and Benning, C. (2018a). Advanced genetic tools enable synthetic biology in the oleaginous microalgae Nannochloropsis sp. Plant Cell Rep. 37, 1383-1399. doi: 10.1007/s00299-018-2270-0

Poliner, E., Takeuchi, T., Du, Z. Y., Benning, C., and Farre, E. M. (2018b). Nontransgenic marker-free gene disruption by an episomal CRISPR system in the Oleaginous Microalga, Nannochloropsis oceanica CCMP1779. ACS Synth. Biol. 7, 962-968. doi: 10.1021/acssynbio.7b00362

Polle, J. E. W., Barry, K., Cushman, J., Schmutz, J., Tran, D., Hathwaik, L. T., et al. (2017). Draft nuclear genome sequence of the halophilic and betacarotene-accumulating green alga Dunaliella salina strain CCAP19/18. Genome Announc. 5:e01105-17. doi: 10.1128/genomeA.01105-17

Pourmir, A., and Johannes, T. W. (2012). Directed evolution: selection of the host organism. Comput. Struct. Biotechnol. J. 2:e201209012. doi: 10.5936/csbj.201209012

Rossoni, A. W., and Weber, A. P. M. (2019). Systems biology of cold adaptation in the polyextremophilic red alga Galdieria sulphuraria. Front. Microbiol. 10:927. doi: $10.3389 /$ fmicb.2019.00927

Scaife, M. A., Nguyen, G., Rico, J., Lambert, D., Helliwell, K. E., and Smith, A. G. (2015). Establishing Chlamydomonas reinhardtii as an industrial biotechnology host. Plant J. 82, 532-546. doi: 10.1111/tpj.12781

Schaart, J. G., Van De Wiel, C. C. M., and Smulders, M. J. M. (2021). Genome editing of polyploid crops: prospects, achievements and bottlenecks. Transgenic Res. 30, 337-351. doi: 10.1007/s11248-021-0 0251-0

Sharma, A. K., Nymark, M., Sparstad, T., Bones, A. M., and Winge, P. (2018). Transgene-free genome editing in marine algae by bacterial conjugationcomparison with biolistic CRISPR/Cas9 transformation. Sci. Rep. 8:14401. doi: 10.1038/s41598-018-32342-0

Sharma, P. K., Goud, V. V., Yamamoto, Y., and Sahoo, L. (2021). Efficient Agrobacterium tumefaciens-mediated stable genetic transformation of green microalgae, Chlorella sorokiniana. 3 Biotech 11:196. doi: $10.1007 /$ s13205-021-02750-7

Siaut, M., Heijde, M., Mangogna, M., Montsant, A., Coesel, S., Allen, A., et al. (2007). Molecular toolbox for studying diatom biology in Phaeodactylum tricornutum. Gene 406, 23-35. doi: 10.1016/j.gene.2007.05.022

Song, G., Wang, W., Hu, L., Liu, Y., Li, A., Du, J., et al. (2019). An exploration of the rapid transformation method for Dunaliella salina system. AMB Express 9:181. doi: 10.1186/s13568-019-0 905-3

Srivastava, R. K., Bollam, S., Pujarula, V., Pusuluri, M., Singh, R. B., Potupureddi, G., et al. (2020). Exploitation of heterosis in pearl millet: a review. Plants 9:807. doi: $10.3390 /$ plants 9070807

Suttangkakul, A., Sirikhachornkit, A., Juntawong, P., Puangtame, W., Chomtong, T., Srifa, S., et al. (2019). Evaluation of strategies for improving the transgene expression in an oleaginous microalga Scenedesmus acutus. BMC Biotechnol. 19:4. doi: 10.1186/s12896-018-0497-z

Tanaka, T., Maeda, Y., Veluchamy, A., Tanaka, M., Abida, H., Marechal, E., et al. (2015). Oil accumulation by the oleaginous diatom Fistulifera solaris as revealed by the genome and transcriptome. Plant Cell 27, 162-176. doi: 10.1105/tpc.114.135194

Vieler, A., Wu, G., Tsai, C. H., Bullard, B., Cornish, A. J., Harvey, C., et al. (2012). Genome, functional gene annotation, and nuclear transformation of the heterokont oleaginous alga Nannochloropsis oceanica CCMP1779. PLoS Genet. 8:e1003064. doi: 10.1371/journal.pgen.1003064

Wang, K., Cui, Y., Wang, Y., Gao, Z., Liu, T., Meng, C., et al. (2020). Chloroplast genetic engineering of a unicellular green alga Haematococcus pluvialis with expression of an antimicrobial peptide. Mar. Biotechnol. 22, 572-580. doi: 10.1007/s10126-020-09978-z

Wetherell, D. F., and Krauss, R. W. (1956). Colchicine-induced polyploidy in Chlamydomonas. Science 124, 25-26. doi: 10.1126/science.124.3210.25

Wilson, N. F. (2008). Gametic cell adhesion and fusion in the unicellular alga Chlamydomonas. Methods Mol. Biol. 475, 39-51. doi: 10.1007/978-1-59745-250-2_3

Wu, T., Li, L., Jiang, X., Yang, Y., Song, Y., Chen, L., et al. (2019). Sequencing and comparative analysis of three Chlorella genomes provide insights into strain-specific adaptation to wastewater. Sci. Rep. 9:9514. doi: 10.1038/s41598-019-45511-6

Yuan, G., Xu, X., Zhang, W., Zhang, W., Cui, Y., Qin, S., et al. (2019). Biolistic Transformation of Haematococcus pluvialis with constructs based on the flanking sequences of its endogenous alpha tubulin gene. Front. Microbiol. 10:1749. doi: $10.3389 /$ fmicb. 2019.01749

Conflict of Interest: The author declares that the research was conducted in the absence of any commercial or financial relationships that could be construed as a potential conflict of interest.

Publisher's Note: All claims expressed in this article are solely those of the authors and do not necessarily represent those of their affiliated organizations, or those of the publisher, the editors and the reviewers. Any product that may be evaluated in this article, or claim that may be made by its manufacturer, is not guaranteed or endorsed by the publisher.

Copyright $\odot 2021$ Maréchal. This is an open-access article distributed under the terms of the Creative Commons Attribution License (CC BY). The use, distribution or reproduction in other forums is permitted, provided the original author(s) and the copyright owner(s) are credited and that the original publication in this journal is cited, in accordance with accepted academic practice. No use, distribution or reproduction is permitted which does not comply with these terms. 